\title{
Partial duality and closed 2-cell embeddings
}

\author{
To Adrian Bondy on his 70th birthday
}

\author{
M. N. Ellingham ${ }^{1,3}$ \\ Department of Mathematics, 1326 Stevenson Center \\ Vanderbilt University, Nashville, Tennessee 37240, U.S.A. \\ mark. ellingham@vanderbilt.edu \\ Xiaoya Zha ${ }^{2,3}$ \\ Department of Mathematical Sciences, Box 34 \\ Middle Tennessee State University \\ Murfreesboro, Tennessee 37132, U.S.A. \\ xiaoya.zha@mtsu.edu \\ April 28, 2016; to appear in Journal of Combinatorics
}

\begin{abstract}
In 2009 Chmutov introduced the idea of partial duality for embeddings of graphs in surfaces. We discuss some alternative descriptions of partial duality, which demonstrate the symmetry between vertices and faces. One is in terms of band decompositions, and the other is in terms of the gem (graph-encoded map) representation of an embedding. We then use these to investigate when a partial dual is a closed 2 -cell embedding, in which every face is bounded by a cycle in the graph. We obtain a necessary and sufficient condition for a partial dual to be closed 2-cell, and also a sufficient condition for no partial dual to be closed 2-cell.
\end{abstract}

\section{Introduction}

In this paper a surface $\Sigma$ means a connected compact 2-manifold without boundary. By an open or closed disk in $\Sigma$ we mean a subset of the surface homeomorphic to such a subset of $\mathbb{R}^{2}$. By a simple closed curve or circle in $\Sigma$ we mean an image of a circle in $\mathbb{R}^{2}$ under a continuous injective map; a simple arc is a similar image of $[0,1]$. The closure of a set $S$ is denoted $\bar{S}$, and the boundary is denoted $\partial S$.

All our graphs are finite and may (and frequently do) have multiple edges or loops. An embedding of a graph in a surface maps each vertex to a point and each edge to a simple arc or simple closed curve, so that the only pairwise intersections of those points and arcs correspond to the incidences between an edge and its endvertices. For convenience, we usually do not distinguish between a vertex or edge of a graph and its image in a graph embedding. A face of a graph embedding is a connected component of the topological subspace of the surface obtained by deleting the images of all vertices and edges. If two embeddings of a graph are related by a homeomorphism of the surface we consider them to be equivalent. We typically use $G$ to denote not just a graph, but a graph embedding, with vertex set $V(G)$, edge set $E(G)$ and face set $F(G)$; $\Gamma(G)$ denotes the underlying graph.

A graph embedding in a surface is 2-cell, open 2-cell or cellular if every face is an open disk. A 2-cell embedding is a closed 2-cell embedding if every face is bounded by a simple closed curve; equivalently, the boundary walk of each face is a cycle in the graph. The graph is necessarily nonseparable, so if it has at least

\footnotetext{
${ }^{1}$ Supported by National Security Agency grant H98230-13-1-0233 and Simons Foundation award 245715.

${ }^{2}$ Supported by National Security Agency grant H98230-13-1-0216.

${ }^{3}$ The United States Government is authorized to reproduce and distribute reprints notwithstanding any copyright notation herein.
} 
two edges it has no loops and no cutvertices. (There is some ambiguity in the definition of 'closed 2-cell' in the literature. The definition we use is the one given by Barnette 1 in what seems to be the first mention of the concept. Another common definition is that the closure of every face is a closed disk; this is not quite the same, because it allows the boundary of a face, considered as a subgraph, to be a cycle with attached trees. For 2-connected graphs the two definitions are equivalent. Our graphs, however, are not necessarily 2-connected so it is important to be clear about which definition we are using.)

Because all faces in a 2-cell embedding are homeomorphic to an open disk, we can describe such an embedding without giving topological information for each face. In fact, it is well known that 2-cell embeddings can be described in a completely combinatorial way, using a rotation system with edge types or edge signatures. See [14, Section 3.2] and [16, Sections 3.2, 3.3]. A 2-cell embedding can also be described in a number of other ways, including as a band decomposition or as a reduced band decomposition, also called a ribbon graph. We assume the reader is familiar with these different representations of graph embeddings.

For the remainder of this paper all embeddings will be 2-cell embeddings of graphs in surfaces. We will sometimes mention this explicitly, but often just assume it implicitly. Since our surfaces are connected, these are necessarily embeddings of connected graphs.

It is well known that for every 2-cell graph embedding $G$ there is a unique dual graph embedding $G^{*}$ (sometimes called the geometric dual) in the same surface, obtained as follows. Insert one vertex of $G^{*}$ in each face of $G$, and for each edge $e$ of $G$ add an edge $e^{*}$ in $G^{*}$, crossing $e$, which joins the vertices of $G^{*}$ corresponding to the faces on either side of $e$. Then $G^{*}$ is also 2-cell, the faces of $G^{*}$ correspond to the vertices of $G$ and vice versa, and $\left(G^{*}\right)^{*}=G$. Since there is a bijection $e \leftrightarrow e^{*}, e$ and $e^{*}$ are often thought of as the same object. We will distinguish between them only when necessary (usually when considering them as actual curves in a surface).

There are other duals for graph embeddings. For example, the Petrie dual is formed by toggling the signature of every edge (from +1 to -1 , or vice versa) in a representation of the embedding in terms of rotation systems and edge signatures. It is clear that it is possible to do this in a partial fashion, by toggling the signatures for some subset of edges, instead of for all edges. Taking the partial Petrie dual is a useful operation. For example, the process used by the authors in 10 to convert projective-planar embeddings into orientable closed 2-cell embeddings, although described in that paper as a multistep process of inserting crosscaps, then cutting through them and capping off, is really just taking partial Petrie duals.

No one, however, seems to have considered a partial version of geometric duality for graph embeddings, until Chmutov [ $[\underline{6}$ in 2009. He was motivated the desire to unify several Thistlethwaite-type theorems; results of this type relate polynomials for knots or links to graph polynomials. He defines the partial dual, with respect to a subset of edges, for a signed graph, where each edge is assigned either + or - . His main result is an invariance formula for a certain specialization of the Bollobás-Riordan polynomial of signed graphs under partial duality. The Bollobás-Riordan polynomial is formulated in terms of ribbon graphs [3]. Chmutov's original definition of partial duality therefore used ribbon graphs, and another related representation for 2-cell embeddings, which he calls an arrow presentation.

Our interest is purely in the topological structure of a graph embedding under the partial duality operation, and we therefore ignore the part of Chmutov's operation that involves changing the signs of edges, and just deal with unsigned graphs. Several alternative constructions for Chmutov's partial duality operation have appeared in the literature, and are summarized in [12, Section 2.2]. All of these are based on ribbon graphs and arrow presentations. We will provide details of one of these constructions in Section 2

Section 2 discusses two alternative representations of partial duality that do not use ribbon graphs or arrow presentations. The first uses the band decomposition representation of an embedding. The second uses a completely combinatorial representation of a graph embedding as a graph-encoded map, or gem. Both of these definitions give explicit descriptions of the faces as well as the vertices of the partial dual, and demonstrate symmetry with respect to vertices and faces.

In Section 3 we consider the question of whether a partial dual is closed 2-cell. We obtain a necessary and sufficient condition for a partial dual of a general graph embedding to be closed 2-cell. This has a particularly simple form for initial embeddings that are closed 2-cell and satisfy certain additional properties. We also obtain a sufficient condition for no partial dual to be closed 2-cell.

Before we begin we offer an apology of sorts to the reader. Much of our work is just manipulating definitions, and converting ideas from one representation of an embedding into another. We work with many different ways of looking at an embedding, and many related but not identical concepts, and the reader may find this confusing. Unfortunately, this seems to be necessary to get anywhere in this area: different representations are useful for different purposes. We have tried to keep the notation as consistent as possible, 
to help the reader follow what is going on.

\section{Partial duality for band decompositions and gems}

In this section we discuss two representations of partial duality. Graph embeddings can be described in many ways, and so there are also many ways to describe partial duality. The motivation of the two descriptions we give here is that they enable our work in Section 3, characterizing closed 2-cell partial duals. The description in terms of band decompositions (Proposition 2.2 is convenient in situations where we deal with an actual embedding, rather than a model such as a ribbon graph. We specialize this to a particular type of band decomposition which we call a corner graph. The description in terms of gems (Proposition 2.3) is very simple. We provide examples which we hope will be helpful for understanding.

We briefly remind the reader how band decompositions and ribbon graphs of a graph embedding $G$ are found; for a complete description see [14, Subsection 3.2.1]. For the band decomposition we 'fatten' both vertices and edges into closed disks, known as 0-bands for the vertices, and 1-bands for the edges; each face then also corresponds to a closed disk, called a 2-band. A reduced band decomposition or ribbon graph representation may then be obtained by discarding the 2-bands.

For adjacent edges of $G$, their 1-bands may or may not intersect, at a point. We can modify a band decomposition so that they always intersect, or always do not intersect. To allow adjacent 1-bands to intersect when they are the same band, we loosen the definition of a 1-band a little by allowing it to be a closed disk with two points on its boundary identified, or a closed disk with two pairs of points $p_{1}$ and $p_{2}$, $p_{3}$ and $p_{4}$ identified, where $p_{1}, p_{2}, p_{3}, p_{4}$ appear in that order around the boundary.

Standard description of a band decomposition or ribbon graph. We will describe a band decomposition or ribbon graph of a graph embedding $G$ in the following standard way. The 0-bands will be denoted $\overline{c_{v}}$ for $v \in V(G) ; c_{v}$ is the interior of the band. Similarly the 1-bands will be denoted $\overline{q_{e}}$ for $e \in E(G)$, and the 2-bands (if we have them) will be denoted $\overline{a_{f}}$ for $f \in F(G)$. The boundaries of the bands in a band decomposition $N$ of a connected graph embedding with at least one edge may be regarded as an embedded graph $\Theta(N)$ : the vertices are points where three or four bands (one 0-band, one 2-band, and one or two 1-bands) meet, the boundary segments between these points define the edges, and the interiors of the bands are the faces. In $\Theta(N)$, every face $c_{v}$ is bounded by a cycle $C_{v}$, every face $a_{f}$ is bounded by a cycle $A_{f}$, and every face $q_{e}$ is bounded by a closed trail (no repeated edges) $Q_{e}$ of length four, which is a cycle except possibly when $e$ is incident in $G$ with a vertex or face of degree one. If $O$ is a ribbon graph we form a band decomposition $N$ by gluing a 2-band along every boundary component, and then define $\Theta(O)=\Theta(N)$.

We let $C=\bigcup_{v \in V(G)} C_{v}$ and $A=\bigcup_{f \in F(G)} A_{f}$. For any $D \subseteq E(G)$ we let $Q_{D}=\bigcup_{e \in D} Q_{e}, C_{D}=C \cap Q_{D}$ and $A_{D}=A \cap Q_{D}$. The edges of each $Q_{e}$ alternate between edges of $C$ and of $A$.

The following may help the reader remember the notation above. We can think of colouring the edges of $\Theta(N)$, generalizing the standard colouring of edges in a graph-encoded map, which we discuss later in this section. An edge between 0- and 1-bands is red, an edge between 0- and 2-bands is yellow, and an edge between 1- and 2-bands is blue. We use this colour coding consistently in our figures. The boundary of a 0-band contains red edges and possibly some yellow edges, so we use the letter $C$ for crimson, a type of red. The boundary of a 2-band contains blue edges and possibly some yellow edges, so we use the letter $A$ for azure, a type of blue. (We cannot use $R$ and $B$ because we use those later for edges in a graph-encoded map.) The boundary of a 1-band is a quadrilateral (with alternating red and blue edges), so we use the letter $Q$.

The following description of partial duals is convenient for us because it uses only ribbon graphs; it does not require arrow presentations.

Proposition 2.1 (Bradford, Butler and Chmutov [5, Definition 1.2]). Given a connected graph embedding $G$ represented by a ribbon graph $O$, and $D \subseteq E(G)$, we may construct a ribbon graph $O^{D}$ corresponding to $G^{D}$, the partial dual of $G$ with respect to $D$, as follows.

(1) Take the (possibly disconnected) ribbon subgraph $\Delta$ of $O$ consisting of bands $\overline{c_{v}}$ for $v \in V(G)$ and $\overline{q_{e}}$ for $e \in D$ (using our standard description above). Let the boundary of $\Delta$ be $C_{1}^{\prime} \cup C_{2}^{\prime} \cup \ldots \cup C_{k}^{\prime}$, where each $C_{i}^{\prime}$ is a simple closed curve, representing a cycle in $\Theta(O)$.

(2) Add to $O$ a new 0 -band $\overline{c_{i}^{\prime}}$ with boundary $C_{i}^{\prime}$ for $i=1,2, \ldots, k$. Then remove every 0 -band interior $c_{v}$ for $v \in V(G)$ to give $O^{D}$. 
For our purposes the difficulty with this description of partial duality, or other descriptions involving ribbon graphs and arrow presentations, is that it does not explicitly describe the faces of the partial dual. The vertices of $G^{D}$ are explicitly determined by $C_{1}^{\prime} \cup C_{2}^{\prime} \cup \ldots C_{k}^{\prime}$, but the faces are determined only implicitly. For our results on closed 2-cell embeddings in Section 3 we need an explicit description of both vertices and faces. The problem is that ribbon graphs themselves do not explicitly describe faces, since we discard the 2-bands.

We use $S \oplus T$ to denote the symmetric difference of two sets, $(S-T) \cup(T-S)$.

Proposition 2.2. Given a connected graph embedding $G$ in $\Sigma$ represented by a band decomposition $N$ of $\Sigma$, and $D \subseteq E(G)$, we may construct a band decomposition $N^{D}$ corresponding to $G^{D}$, the partial dual of $G$ with respect to $D$, as follows, using our standard decomposition for $N$.

(1) Take the (possibly disconnected) subspace $\Delta$ of $\Sigma$ that is the union of $\overline{c_{v}}$ for all $v \in V(G)$ and $\overline{q_{e}}$ for all $e \in D$. Let the boundary of $\Delta$ be $C^{\prime}=C_{1}^{\prime} \cup C_{2}^{\prime} \cup \ldots \cup C_{k}^{\prime}$, where each $C_{i}^{\prime}$ is a simple closed curve, representing a cycle in $\Theta(N)$.

(2) Let $P=E(G)-D$ and take the (possibly disconnected) subspace $\Pi$ of $\Sigma$ that is the union of $\overline{c_{v}}$ for all $v \in V(G)$ and $\overline{q_{e}}$ for all $e \in P$. Let the boundary of $\Pi$ be $A^{\prime}=A_{1}^{\prime} \cup A_{2}^{\prime} \cup \ldots \cup A_{\ell}^{\prime}$, where each $A_{j}^{\prime}$ is a simple closed curve, representing a cycle in $\Theta(N)$.

(3) Delete from $N$ all 0-band interiors $c_{v}$ for $v \in V(G)$ and all 2-band interiors $a_{f}$ for $f \in F(G)$, leaving the union of $\Theta(N)$ and all $\overline{q_{e}}$ for $e \in E(G)$. Glue on new 0 -bands $\overline{c_{i}^{\prime}}$ with $\partial c_{i}^{\prime}=C_{i}^{\prime}$ for $i=1,2, \ldots, k$, and new 2-bands $\overline{a_{j}^{\prime}}$ with $\partial a_{j}^{\prime}=A_{j}^{\prime}$ for $j=1,2, \ldots, \ell$. The result is $N^{D}$.

Proof. Form a ribbon graph $O$ by discarding the 2-bands of $N$. We can obtain a band decomposition of $G^{D}$ by adding 2-bands along the boundary of $O^{D}$ from Proposition 2.1. The 0-bands and 1-bands of $N^{D}$ as constructed here are identical to those for $O^{D}$ from Proposition 2.1, so we just need to show that the boundaries of the 2-bands of $N^{D}$, which form $\partial \Pi$, are the same as the boundary of $O^{D}$, i.e., that $\partial \Pi=\partial\left(O^{D}\right)$.

To avoid cumbersome notation, all equations in this paragraph are correct up to adding or deleting finitely many points (which will be vertices of $\Theta(N)$ ); this is sufficient to prove our conclusion. Since $\Delta=\left(\bigcup_{v \in V(G)} \overline{c_{v}}\right) \cup\left(\bigcup_{e \in D} \overline{q_{e}}\right)$ we have $\partial \Delta=\left(\bigcup_{v \in V(G)} C_{v}\right) \oplus\left(\bigcup_{e \in D} Q_{e}\right)=C \oplus Q_{D}$. Similarly, $\partial \Pi=$ $C \oplus Q_{P}$. Now $O^{D}=\left(\bigcup_{i=1}^{k} \overline{c_{i}^{\prime}}\right) \cup\left(\bigcup_{e \in E(G)} \overline{q_{e}}\right)$ so that $\partial\left(O^{D}\right)=\left(\bigcup_{i=1}^{k} C_{i}^{\prime}\right) \oplus\left(\bigcup_{e \in E(G)} Q_{e}\right)=\partial \Delta \oplus Q=$ $\left(C \oplus Q_{D}\right) \oplus Q=C \oplus\left(Q \oplus Q_{D}\right)=C \oplus Q_{P}=\partial \Pi$, as required.

As pointed out by one of the referees, the claim that $\partial \Pi=\partial\left(O^{D}\right)$ in the above proof can also be proved using basic properties of partial duality for ribbon graphs established by Chmutov 6 .

When we are forming a dual $G^{D}$ we refer to the elements of $D$ as dual edges and the elements of $E(G)-D$ as primal edges. In Proposition 2.2, loosely $\Delta$ is the region of the surface $\Sigma$ containing all vertices and the dual edges, and its boundary $C^{\prime}=\partial \Delta$ specifies the new vertices. Similarly, $\Pi$ is the region of the surface containing all vertices and the primal edges, and its boundary $A^{\prime}=\partial \Pi$ specifies the new faces. We identify the new vertex associated with $C_{i}^{\prime}$ as $v_{i}^{\prime}$, and the new face associated with $A_{j}^{\prime}$ as $f_{j}^{\prime}$.

The construction of Proposition 2.2 explicitly describes the faces (via 2-bands), as well as the vertices (via 0-bands), of $G^{D}$. This construction also shows that partial duality treats dual and primal edges in some sense symmetrically. Moreover, the union of the bands not in $\Pi$, namely $\overline{a_{f}}$ for all $f \in F(G)$, and $\overline{q_{e}}$ for all $e \in D$, forms a region $\Delta^{*}$ complementary to $\Pi$ (ignoring overlap along the boundary), so $\partial \Delta^{*}=\partial \Pi$. Therefore, the above description can also be expressed in a way that treats faces and vertices symmetrically, with $C^{\prime}=\partial \Delta$ and $A^{\prime}=\partial \Delta^{*}$.

It is helpful to remove the ambiguity in the definition of band decomposition as to whether 1-bands can intersect. For our main result in Section 3 , we will use a band decomposition where 1-bands corresponding to adjacent edges always intersect. To help familiarize the reader with this, we use it as the basis for our example to illustrate Proposition 2.2 .

We first construct the barycentric subdivision $S$ of a graph embedding $G$ (see [16, p. 154]), which we will use again later. We replace each edge $e$ by a path of length two through a new vertex $u_{e}$, then add a new vertex $u_{f}$ in each face $f$, joined to all vertices (including new vertices $u_{e}$ ) on the boundary of $f$. Let $U_{0}=V(G), U_{1}=\left\{u_{e} \mid e \in E(G)\right\}$ and $U_{2}=\left\{u_{f} \mid f \in F(G)\right\}$. Then $S$ is a triangulation, where each triangle contains one vertex from each of $U_{0}, U_{1}$ and $U_{2}$. Let $E_{j k}, 0 \leq j<k \leq 2$, be the set of edges between $U_{j}$ and $U_{k}$ in $S$. The radial graph $S_{02}$ is the subgraph of $S$ induced by $U_{0} \cup U_{2}$; its edge set is $E_{02}$. Define a corner to be the midpoint of an edge of $E_{02}$. Every corner $y$ is associated with a vertex $\nu(y)$ of $G$, a face $\phi(y)$ of $G$, 


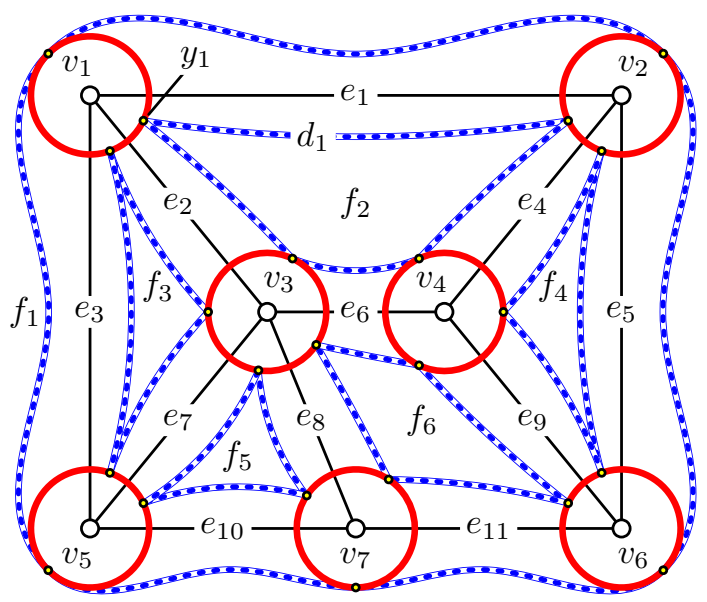

Figure 1: Graph embedding and its corner graph

and with an unordered pair $\varepsilon_{G}(y)$ of (possibly equal) edges of $G$. It is also associated with an edge $\varepsilon_{S}(y)$ of $S$. A corner can be thought of as marking the place where a vertex, a face and two edges of $G$ come together.

The corner graph $K$ is a graph embedding with the corners of $G$ as its vertices. For edges we add a cycle $C_{v}$ of length $\operatorname{deg}_{G}(v)$ around each vertex $v$ of $G$, through the corners of $v$ in their order around $v$, and a cycle $A_{f}$ of length $\operatorname{deg}_{G}(f)$ inside each face $f$ of $G$, through the corners of $f$ in their order around $f$. Then $K$ has three types of face: each $v \in V(G)$ is in a face $c_{v}$ with $\partial c_{v}=C_{v}$, each $f \in F(G)$ contains a face $a_{f}$ with $\partial a_{f}=A_{f}$, and each $e \in E(G)$ with endvertices $u$ and $v$ intersects a unique face $q_{e}$ of degree 4 in addition to $c_{u} \cup c_{v}$. Each vertex of $K$ has degree $4, C=\bigcup_{v \in V(G)} C_{v}$ and $A=\bigcup_{f \in F(G)} A_{f}$ are edge-disjoint 2-factors of $K$ with $C \cup A=K$, and $\left\{Q_{e} \mid e \in E(G)\right\}$ is a decomposition of $K$ into edge-disjoint closed trails of length 4 (so that $Q=\bigcup_{e \in E(G)} Q_{e}=K$ ). The closures of the faces of $K$ give a band decomposition of $G$.

Each edge $d$ of $K$ is associated with a unique edge $\varepsilon_{G}(d)=e$ of $G$ by $d \in E\left(Q_{e}\right)$, so $d$ either parallels or crosses $e$. We can also define a function $\chi(d)$ for $d \in E(K)$, which takes the value 1 if $d$ crosses $\varepsilon_{G}(d)$ in $\Sigma$, and 0 when $d$ parallels $\varepsilon_{G}(d)$. In other words, $\chi(d)=1$ if $d \in E(C)$, and 0 if $d \in E(A)$.

If we dualize a set of edges $D$ in $G$, then from Proposition 2.2 the corner graph $K^{D}$ corresponding to the partial dual $G^{D}$ has the same underlying graph as $K$. We also keep the same 1-bands, but get new 0-bands and 2-bands using Proposition 2.2 .

We illustrate this using Figure 1. where we see a plane graph $G$ (thin lines) and its corner graph $K$, with edges of $C$ shown in red (solid) and edges of $A$ shown in blue (dashed). Each face of $K$ is labelled by a vertex, edge or face of $G$. For the labelled vertex $y_{1}$ of $K$, we have $\nu\left(y_{1}\right)=v_{1}, \phi\left(y_{1}\right)=f_{2}$, and $\varepsilon_{G}\left(y_{1}\right)=\left\{e_{1}, e_{2}\right\}$. For the labelled edge $d_{1}$ of $K$, we have $\varepsilon_{G}\left(d_{1}\right)=e_{1}$, and $\chi\left(d_{1}\right)=0$, since $d_{1}$ does not cross $e_{1}$.

Now suppose we form $G^{D}$ where $D=\left\{e_{2}, e_{3}, e_{4}, e_{7}, e_{10}\right\}$; edges of $D$ are shown as wavy edges in Figure 2. We now change the colours of the edges of $K$ corresponding to $D$ to describe the new vertices and faces of $K^{D}$. The boundary components of $\partial \Delta$, providing new vertices, are shown in red (solid); these form $C^{\prime}=C_{1}^{\prime} \cup C_{2}^{\prime} \cup C_{3}^{\prime} \cup C_{4}^{\prime}$, enclosing regions containing the edges of $D$. The boundary components of $\partial \Pi$, providing new faces, are shown in blue (dashed); these form $A^{\prime}=A_{1}^{\prime} \cup A_{2}^{\prime} \cup A_{3}^{\prime}$, enclosing regions containing the edges of $P=\left\{e_{1}, e_{5}, e_{6}, e_{8}, e_{9}, e_{11}\right\}$.

The edges incident with a new vertex or face, and their order, are determined by applying $\varepsilon_{G}$ to the edges of the appropriate cycle $C_{i}^{\prime}$ or $A_{j}^{\prime}$. For example, new vertex $v_{3}^{\prime}$ corresponds to $C_{3}^{\prime}$, and tracing around $C_{3}^{\prime}$ clockwise we see that we parallel or cross edges $e_{1}, e_{5}, e_{4}, e_{9}, e_{6}$ and $e_{4}$ again. That is the order of edges around $v_{3}^{\prime}$. The new face $f_{2}^{\prime}$ corresponds to $A_{2}^{\prime}$, and tracing around that we see that we cross edges $e_{3}, e_{7}$ and $e_{10}$. That is the order of edges around $f_{2}^{\prime}$. Chmutov [6] showed that a partial dual of an orientable embedding is always orientable. So knowing the rotation around each vertex, we can construct the partial dual embedding. In the nonorientable case, we must be more careful; we need to know how the edges of a cycle $Q_{e}$, corresponding to edges, are traversed by the corresponding new vertices, so we can decide whether the corresponding edge in the dual is twisted (signature -1) or not. That is the information carried by the arrow presentation in Chmutov's original formulation of partial duality; in Propositions 2.1 and 2.2 it is carried by the faces $\overline{q_{e}}$, which we never delete.

We also consider a band decomposition where all 1-bands are pairwise disjoint. We again base it on the 


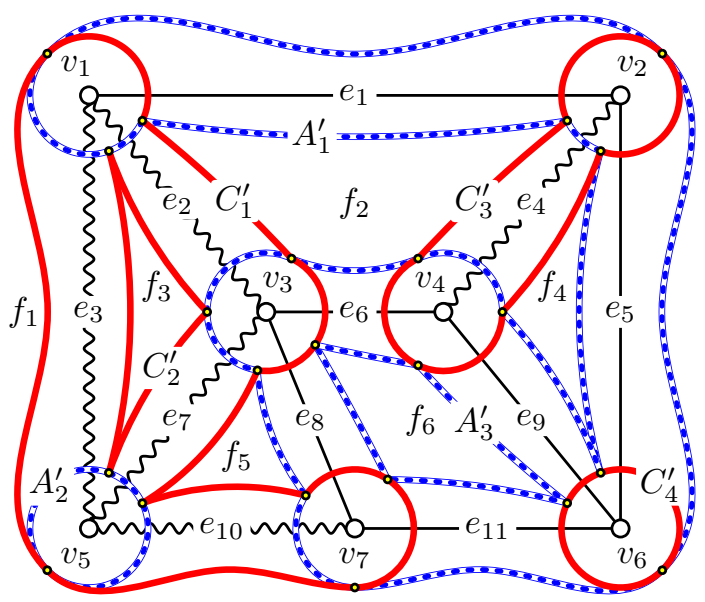

Figure 2: Dualized corner graph

barycentric subdivision $S$, using the notation developed above. Since $S$ is a triangulation, its dual $S^{*}$ is a cubic graph embedding where each vertex is incident with exactly one edge from each of $E_{01}^{*}, E_{02}^{*}$ and $E_{12}^{*}$, where $E_{j k}^{*}$ is the set of dual edges for $E_{j k}$ (here we distinguish between edges and their duals). If $U_{j}^{*}$ denotes the set of faces of $S^{*}$ dual to the vertices in $U_{j}$, then the closure of each element of $U_{j}^{*}$ is a $j$-band in a band decomposition of $G$ in which 1-bands are pairwise disjoint, with $C=\bigcup_{c \in U_{0}^{*}} \partial c, Q=\bigcup_{q \in U_{1}^{*}} \partial q$ and $A=\bigcup_{a \in U_{2}^{*}} \partial a$.

Colour the edges of $S^{*}$ so that edges of $E_{01}^{*}$ are red, of $E_{02}^{*}$ are yellow, and of $E_{12}^{*}$ are blue. (Our choice of colours here follows 4 and agrees with that in our standard description of a band decomposition, earlier.) This is a proper 3-edge-colouring. In an edge-coloured graph, we define a bigon to be a cycle whose edges alternate between two colours. Each face $c_{v} \in U_{0}^{*}$ is bounded by a red-yellow bigon; each $q_{e} \in U_{1}^{*}$ is bounded by a red-blue bigon; and each $a_{f} \in U_{2}^{*}$ is bounded by a blue-yellow bigon. If we take the underlying graph $\Gamma\left(S^{*}\right)$ and keep the edge colours, we have the graph-encoded map or gem representation $M$ of $G$. We can clearly recover the graph embedding $S^{*}$, and hence $G$, by gluing a disk onto each bigon of $M$.

Although we obtained $M$ from an embedding, it is just an abstract connected, properly 3-edge-coloured, cubic graph. The red-yellow bigons of $M$ correspond to vertices of $G$, the red-blue bigons correspond to edges of $G$ and are all 4-cycles, and the blue-yellow bigons correspond to faces of $G$. Note that $M$ may have parallel edges in red-yellow or blue-yellow pairs, which correspond to vertices and faces of degree one, respectively, but $M$ has no parallel edges in other colour combinations (red-blue, or two of the same colour), and $M$ has no loops.

Gems are a purely combinatorial representation of an embedding. Any properly 3-edge-coloured cubic graph $M$ with colours red, yellow and blue in which the red-blue bigons are 4-cycles is the gem of some embedding. The idea of representing an embedding by a 3-edge-coloured cubic graph appears to be due to Robertson [17, Section 4]. It was developed (appropriately for us) as part of a general theory of graph embedding duality by Lins [15, and used as the basis of a general theory of graph embeddings by Bonnington and Little [4. It has been extended to more general 2-dimensional embeddings, and to representations of higher dimensional objects; see [7].

We now show that partial duality can be expressed very simply in terms of gems. This is a result we have presented in the past (see for example [9]) but this is the first proof we have given in print. Recently Chmutov and Vignes-Tourneret developed a theory of partial duality for hypermaps, which generalize graph embeddings. They have a generalization of Proposition [2.3 in that context [7, Theorem 3.11].

Proposition 2.3. Suppose $G$ is a 2-cell embedding of a connected graph $G$, with gem $M$. Let $D \subseteq E(G)$. Then the partial dual $G^{D}$ corresponds to a gem $M^{D}$ obtained by swapping the colours red and blue on each bigon of $M$ corresponding to an edge in $D$.

Proof. Everything we write here involves sets of edges, but for brevity we just write $H$ instead of $E(H)$, for any subgraph $H$ of $M$.

Let $R=C \cap Q, Y=C \cap A$ and $B=A \cap Q$ be the sets of red, yellow and blue edges in $M$, respectively. Let $R_{D}=R \cap Q_{D}, R_{P}=R \cap Q_{P}, B_{D}=B \cap Q_{D}$ and $B_{P}=B \cap Q_{P}$. Then $R_{D}, R_{P}, B_{D}, B_{P}$ and $Y$ are disjoint 

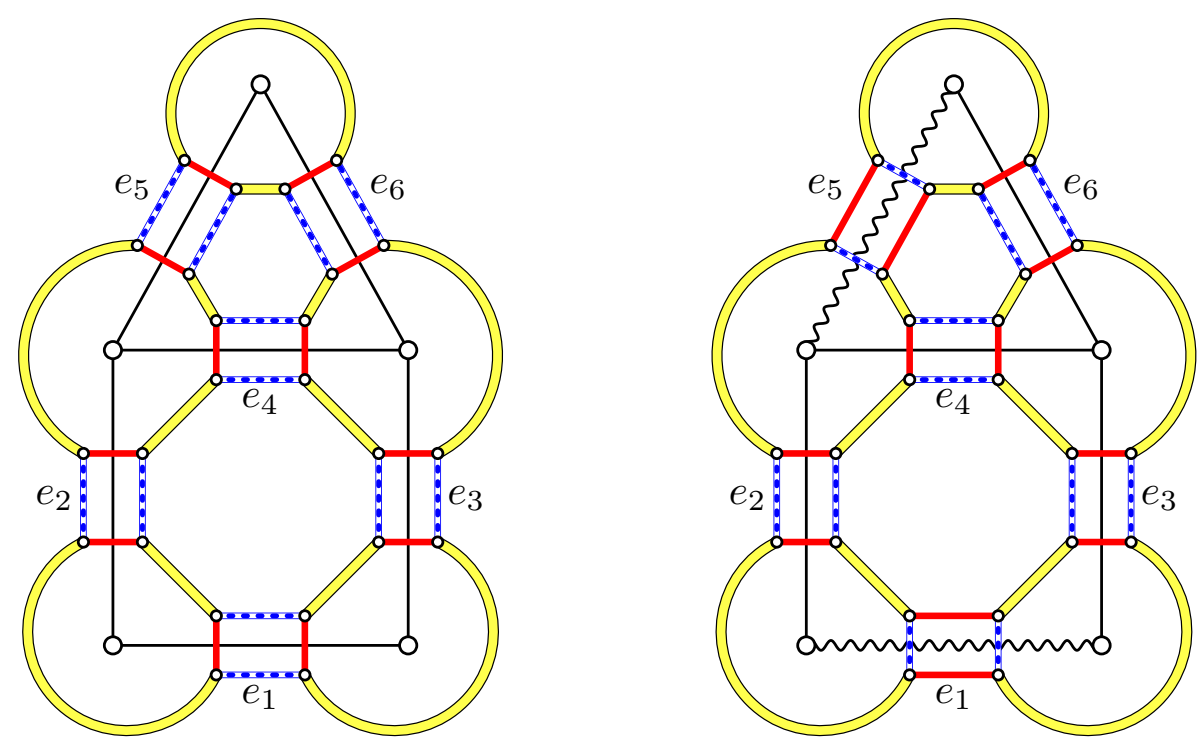

Figure 3: Left: graph and gem; right: after partial dual

and their union is $E(M)$. We have $C=R_{D} \cup R_{P} \cup Y, A=B_{D} \cup B_{P} \cup Y$, and $Q=R_{D} \cup R_{P} \cup B_{D} \cup B_{P}$.

Suppose we take the partial dual; we use results and notation from Proposition 2.2 and its proof. We have $C^{\prime}=C \oplus Q_{D}=\left(R_{D} \cup R_{P} \cup Y\right) \oplus\left(R_{D} \cup B_{D}\right)=R_{P} \cup B_{D} \cup Y$. Similarly, $A^{\prime}=C \oplus Q_{P}=R_{D} \cup B_{P} \cup Y$. And $Q^{\prime}=Q=R_{D} \cup R_{P} \cup B_{D} \cup B_{P}$. Therefore, for $M^{D}, R^{\prime}=C^{\prime} \cap Q^{\prime}=R_{P} \cup B_{D}, Y^{\prime}=C^{\prime} \cap A^{\prime}=Y$, and $B^{\prime}=A^{\prime} \cap Q^{\prime}=R_{D} \cap B_{P}$. In other words, we exchange red and blue on edges in $Q_{D}$, and leave other colours alone, as required.

The description of partial duality in Proposition 2.3 treats red and blue edges, and hence red-yellow and blue-yellow bigons, symmetrically, again illustrating that partial duality treats vertices and faces symmetrically.

We provide an example in Figures 3 and 4. At left in Figure 3 we see a small planar graph and its gem ( with colours indicated as red $=$ solid, blue $=$ dashed, and yellow $=$ hollow). Suppose we dualize $D=\left\{e_{1}, e_{5}\right\}$. At right in Figure 3 we see the original graph, with the dual edges shown as wavy edges, and the new gem. In Figure 4 we have separated out the red-yellow and blue-yellow bigons in the partial dual, and we see that there are now three vertices and only one face.

In the figures, the partial duality operations for gems and for the corner graph seem to be very similar, which is to be expected. The corner graph $K$ can be obtained from the coloured dual of the barycentric subdivision, which is also the embedded gem, by contracting yellow edges, which become the vertices of $K$. Then the red edges of $M$ become $C$ in $K$, and the blue edges of $M$ become $A$ in $K$.

A number of properties of partial duals are very easy to handle using gems. For example, as mentioned earlier, Chmutov showed that the orientability of an embedding is unaltered by taking a partial dual. This follows from the fact that an embedding is orientable if and only if its gem is bipartite [15, pp. 180-181]: this is a fixed property of the underlying graph of the gem, and is not altered by swapping colours on red-blue bigons. Also, Ellis-Monaghan and Moffatt [11, 12 have extensively investigated the interaction between partial duality and partial Petrie duality; they call the combined effect twisted duality. This can be handled using an extension to gems suggested by Lins [15. He added a fourth set of edges to a gem, which we call green edges, adding two diagonals to each red-blue bigon so it becomes a red-blue-green trigon, isomorphic to $K_{4}$. Bonnington and Little [4] call the result a jewel; we show a jewel in Figure 5 (with green indicated by heavy hollow edges). Petrie duality is formulated very naturally in the jewel, as swapping blue and green in red-blue-green trigons: this can obviously be done for a subset of the edges as well as for the whole edge set. Therefore, some aspects of twisted duality are easy to interpret using jewels: for example, twisted duality provides an action of the ribbon group $S_{3}^{E}$ on embeddings with a fixed edge set $E$ [11, Definition 3.4] because we can permute the colours red, blue and green for each $e \in E$. 

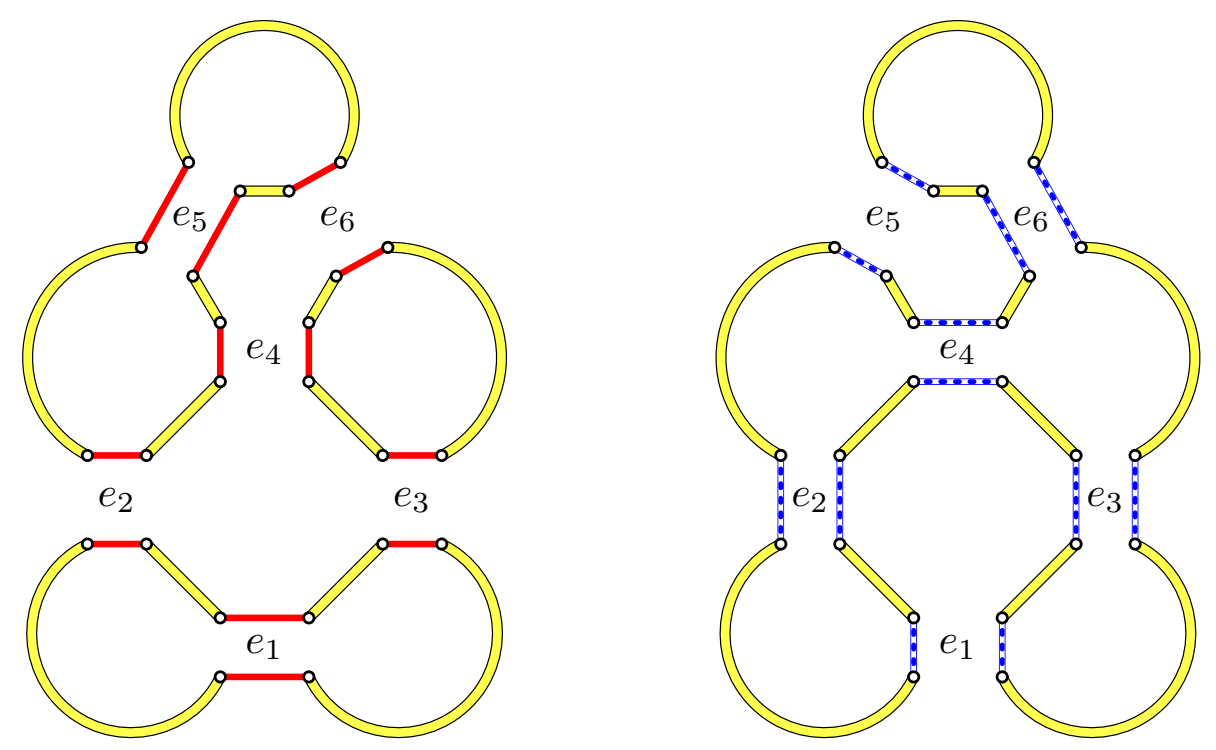

Figure 4: Left: new red-yellow bigons (vertices); right: new blue-yellow bigons (faces)

\section{Closed 2-cell embeddings}

Closed 2-cell embeddings are particularly nice because each face and vertex are incident at most once. They can be regarded as embeddings of nonseparable graphs with facewidth at least 2. The Strong Embedding Conjecture [13] says that every 2-connected graph has a closed 2-cell embedding in some surface. This implies the well-known Cycle Double Cover Conjecture [18, 19, which says that every 2-edge-connected graph has a cycle double cover, i.e., a set of cycles in the graph such that each edge is contained in exactly two of these cycles.

Being closed 2-cell seems to be a property that is quite fragile under taking partial duals. If we start with a closed 2-cell graph embedding, taking the partial dual of any single edge (necessarily a link, or non-loop) creates a loop, and a graph embedding with a loop and at least one other edge is not closed 2-cell. (See [6. Subsection 1.7] or [12, pp. 32-33]. A more general version of this situation is discussed following Lemma 3.2.) However, it is known that the (full geometric) dual of a closed 2-cell embedding is always closed 2-cell. Therefore, we may wonder whether a closed 2-cell embedding has any other closed 2-cell partial duals besides its full dual.

In this section we develop some theory that will allow us to provide examples of closed 2-cell embeddings that have nontrivial partial duals that are closed 2-cell, and to identify certain situations where the partial dual will not be closed 2-cell. To do this we provide a necessary and sufficient condition based on the corner graph for an embedding to be closed 2-cell. Some parts of our condition are unfortunately not easy to check exactly, but stronger versions of these parts are easy to check, so we can obtain useful results. We also use an argument based on gems to say that some graph embeddings have no partial duals that are closed 2-cell.

What prevents an embedding from being closed 2-cell is a bad vertex/face pair $(v, f)$ : a vertex $v$ and face $f$ so that $v$ and $f$ are incident more than once. A good vertex/face pair $(v, f)$ is one where $v$ and $f$ are incident at most once. The definition of a bad vertex/face pair is symmetric in faces and vertices, which is why the full dual of a closed 2-cell embedding is closed 2-cell. Vertex/face incidences occur precisely at the corners of $G$, so these conditions are easy to express using the corner graph $K$ : a bad vertex/face pair occurs if there are some $C_{v}$ and $A_{f}$ that intersect in two or more vertices of $K$. It is also easy to express in terms of the gem: a bad vertex/face pair occurs if there are a red-yellow bigon and a blue-yellow bigon that share two or more yellow edges.

Therefore, for any particular $G$ and $D$, we can use this corner graph condition or gem condition, along with Proposition 2.2 or 2.3 to easily determine whether $G^{D}$ is closed 2-cell. However, we would like to provide some general conclusions. We do this by analyzing Proposition 2.2 in more detail for corner graphs.

Suppose we have a graph embedding $G$ and its corner graph $K$ together in the same surface $\Sigma$. We use the notation of the previous section for band decompositions, including our standard description. These now always refer to the corner graph $K$. Let $D \subseteq E(G)$ and $P=E(G)-D$ be our set of dual and primal edges, 


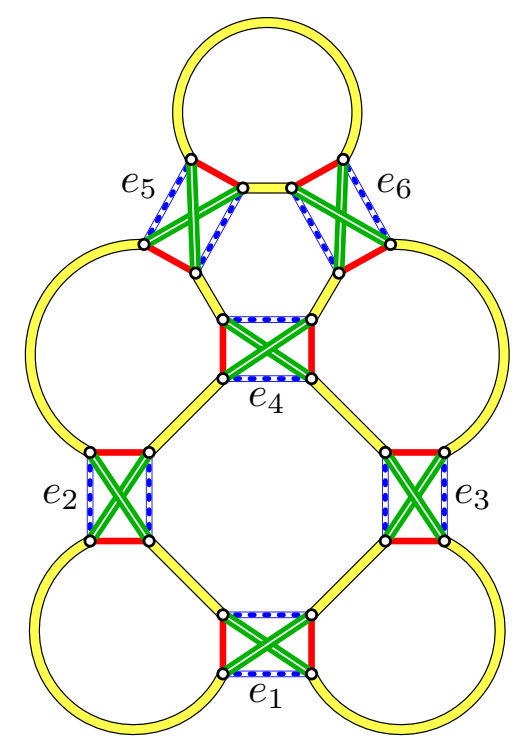

Figure 5: The jewel of our earlier example

respectively.

We now classify elements of $G$ and of $K$ based on the division between primal and dual edges. A vertex or face of $G$ is said to be primal-pure if all of its incident edges belong to $P$, and dual-pure if all of its incident edges belong to $D$. In either case we say it is pure. If at least one incident edge belongs to $P$ and at least one to $D$, then we say a vertex or face of $G$ is mixed. Recall that vertices of $K$ are corners of $G$, and each $y \in V(K)$ has an associated $v=\nu(y) \in V(G), f=\phi(y) \in F(G)$, and $\left\{e_{1}, e_{2}\right\}=\varepsilon_{G}(y)$ which is an unordered pair of (possibly equal) edges of $G$. We say $y$ is $k$-primal-pure ( $k$-dual-pure) if $e_{1}, e_{2} \in P\left(e_{1}, e_{2} \in D\right)$ and exactly $k$ of $v$ or $f$ are pure (so $k=0,1$ or 2). We can drop either the $k$ or the primal/dual distinction, and talk about $k$-pure corners, primal-pure or dual-pure corners, or just pure corners. A corner that is not pure is mixed, which necessarily means that $e_{1} \in D$ and $e_{2} \in P$, or vice versa, and that both $v$ and $f$ are mixed.

We can map every walk $W$ in $K$ to a walk $\omega(W)$ in $G$. Loosely, we project each edge of $W$ that parallels an edge of $G$ to that edge. We ignore edges of $W$ that cross edges of $G$; such edges stay at the same vertex of $G$. More formally, suppose $W$ is represented as an alternating sequence of vertices and edges, $y_{0}, d_{1}, y_{1}, d_{2}, y_{2}, \ldots, y_{m}$ in $K$. We map this sequence to a walk in $G$ as follows: (1) for each $j$, replace vertex $y_{j}$ by $\nu\left(y_{j}\right)$; (2) for each $j$, replace edge $d_{j}$ by $\varepsilon_{G}\left(d_{j}\right)$ if $\chi\left(d_{i}\right)=0$, or delete $d_{i}$ if $\chi\left(d_{i}\right)=1$; (3) now any consecutive subsequences containing only vertices will all be repetitions of the same vertex, so replace them by one copy of that vertex.

From Proposition 2.2 we know that the vertices of $G^{D}$ correspond to components of $C^{\prime}=\partial \Delta$. We can apply $\omega$ to the cycles $C_{i}^{\prime}$. The $\omega\left(C_{i}^{\prime}\right)$ are exactly the components of boundary walks of faces of the (possibly non-2-cell, possibly disconnected) graph embedding obtained from $G$ by deleting edges not in $D$. We keep all vertices of $G$ even if they have no incident edges from $D$; some of the $\omega\left(C_{i}^{\prime}\right)$ may be trivial walks consisting of a single vertex.

In $G$, let $T_{D}$ be the subspace of $\Sigma$ which is the union of $D$ and all dual-pure faces, including all mixed or dual-pure vertices, but no primal-pure vertices. We call $T_{D}$ the totally dual subspace of $\Sigma$, and its components are totally dual regions. Similarly, we let $T_{P}$, the totally primal subspace, be the union of $P$ and all primalpure faces; its components are totally primal regions. We note that $T_{D} \cap T_{P}$ is precisely the set of mixed vertices, and that $\Sigma$ is the disjoint union of $T_{D} \cup T_{P}$ and the mixed faces. The boundary of every mixed face is therefore contained in $\partial T_{P} \cup \partial T_{D}$.

Now each vertex $v_{i}^{\prime}$ of $G^{D}$, can be classified as one of three types, depending on where its corresponding cycle $C_{i}^{\prime}$ came from. First, suppose $C_{i}^{\prime}=C_{v}$ for some $v \in V(G)$. In this case $\omega\left(C_{i}^{\prime}\right)$ will be a trivial walk $v_{i}^{\prime}$. Then $c_{v}$ has no neighbouring faces $q_{e}$ with $e \in D$. In other words, there are no edges of $D$ incident with $v$ in $G$, so $v$ is primal-pure. We write $v_{i}^{\prime} \in V_{\mathrm{ppv}}^{\prime}$. Essentially $v$ and $v_{i}^{\prime}$ are the same vertex, unchanged, and we write $v_{i}^{\prime}=v$. Second, suppose $C_{i}^{\prime}=A_{f}$ for some $f \in F(G)$. In this case $\omega\left(C_{i}^{\prime}\right)=\partial f$. Then all $q_{e}$ adjacent to $a_{f}$ in $K$ must have had $e \in D$. In other words, every edge of $f$ in $G$ belongs to $D$, so $f$ is dual-pure. We write $v_{i}^{\prime} \in V_{\mathrm{dpf}}^{\prime}$. Essentially the face $f$ has been completely dualized to become the vertex $v_{i}^{\prime}$ 


\begin{tabular}{l|r||r|r|r|}
\multicolumn{3}{c}{} & \multicolumn{3}{c}{ face type } \\
\cline { 2 - 5 } \multicolumn{1}{c||}{} & dpv & ppf & tpb \\
\cline { 2 - 5 } vertex & ppv & $\mathrm{X}$ & LC & MC \\
type & dpf & LC & X & MC \\
& tdb & MC & MC & GC \\
\cline { 2 - 6 } & & & &
\end{tabular}

Table 1: Handling bad vertex/face pairs

in $G^{D}$, and we write $v_{i}^{\prime}=f$. To discuss the third type, suppose we expand the region $\Delta$ to $\Delta^{+}$by adding the bands $\overline{A_{f}}$ for all dual-pure faces $f$. The components of $\partial \Delta^{+}$are exactly the components of $\partial \Delta$ that do not come from dual-pure faces; in other words, they come from primal-pure vertices, or are of our third type. But $\Delta^{+}$consists of all bands of $K$ that intersect $T_{D}$, together with bands $\overline{c_{v}}$ for primal-pure vertices $v=v_{i}^{\prime}$. Therefore, for our third type, $C_{i}^{\prime}$ is a boundary cycle for a component of $\Delta^{+}$containing a component $\Phi$ of $T_{D}$, i.e., a totally dual region. The projected walk $\omega\left(C_{i}^{\prime}\right)$ in $G$ is one of the closed walks bounding the region $\Phi$ from the outside ('from the outside' means there is a slight homotopic shifting of $\omega\left(C_{i}^{\prime}\right)$ that does not intersect $\Phi)$. We write $v_{i}^{\prime} \in V_{\mathrm{tdb}}^{\prime}$.

We can apply a similar analysis to faces $f_{j}^{\prime}$ of $G^{D}: f_{j}^{\prime} \in F_{\mathrm{ppf}}^{\prime}$ if it comes from a primal-pure face in $G$; $f_{j}^{\prime} \in F_{\mathrm{dpv}}^{\prime}$ if it comes from a dual-pure vertex in $G ; f_{j}^{\prime} \in F_{\mathrm{tpb}}^{\prime}$ if it comes from a boundary walk of a totally primal region of $G$.

We illustrate these concepts using Figure 2. Cycles $C_{1}^{\prime}$ and $C_{3}^{\prime}$ come from boundary walks of totally dual regions, so $v_{1}^{\prime}, v_{3}^{\prime} \in V_{\mathrm{tdb}}^{\prime}$. Cycle $C_{2}^{\prime}$ is the same as $A_{3}$, so $v_{2}^{\prime}=f_{3} \in V_{\mathrm{dpf}}^{\prime}$. Cycle $C_{4}^{\prime}$ is the same as $C_{6}$, so $v_{4}^{\prime}=v_{6} \in V_{\mathrm{ppv}}^{\prime}$. Cycle $A_{1}^{\prime}$ comes from a boundary walk of a totally primal region, so $f_{1}^{\prime} \in F_{\mathrm{tpb}}^{\prime}$. Cycle $A_{2}^{\prime}$ is the same as $C_{5}$, so $f_{2}^{\prime}=v_{5} \in F_{\mathrm{dpv}}^{\prime}$. Cycle $A_{3}^{\prime}$ is the same as $A_{6}$, so $f_{3}^{\prime}=f_{6} \in F_{\mathrm{ppf}}^{\prime}$.

To eliminate bad vertex/face pairs, therefore, we consider all possible combinations of the three vertex types and three face types in $G^{D}$. Table 1 indicates the way in which we will deal with the nine resulting cases. Here ' $\mathrm{X}$ ' indicates situations that never happen. If $v_{i}^{\prime} \in V_{\mathrm{ppv}}^{\prime}$ and $f_{j}^{\prime} \in F_{\mathrm{dpv}}^{\prime}$ then $C_{i}^{\prime}$ and $A_{j}^{\prime}$ are both cycles of the form $C_{v}$ in $K$, and hence are disjoint, so the corresponding vertex and face of $G^{D}$ are not incident at all, and cannot form a bad pair. Similar reasoning applies when $v_{i}^{\prime} \in V_{\mathrm{dpf}}^{\prime}$ and $f_{j}^{\prime} \in F_{\mathrm{ppf}}^{\prime}$. The remaining situations are handled by three conditions that we call the local condition, the midrange condition and the global condition, represented in the table by LC, MC and GC, respectively.

The local condition is so called because it deals with fixing local problems, namely individual bad pairs in $G$. If there is a bad pair in $G$, we must make sure it does not yield a bad pair in $G^{D}$. In a graph or graph embedding $G$, we denote by $\delta_{G} v$, or just $\delta v$, the set of edges incident with vertex $v$ (including loops at $v$ ).

Lemma 3.1 (Local condition). The following are equivalent:

(i) For every bad vertex/face pair $(v, f)$ in $G$, at least one of $v$ or $f$ is mixed.

(ii) For every bad vertex/face pair $(v, f)$ in $G, \delta v \cup \partial f$ contains both primal and dual edges.

(iii) $G^{D}$ has no bad vertex/face pair $\left(v_{i}^{\prime}, f_{j}^{\prime}\right)$ with $v_{i}^{\prime} \in V_{\mathrm{ppv}}^{\prime}$ and $f_{j}^{\prime} \in F_{\mathrm{ppf}}^{\prime}$, or $v_{i}^{\prime} \in V_{\mathrm{dpf}}^{\prime}$ and $f_{j}^{\prime} \in F_{\mathrm{dpv}}^{\prime}$.

Proof. First we show that (i) $\Leftrightarrow$ (ii). Obviously (i) $\Rightarrow$ (ii). Suppose (ii) holds. Since $(v, f)$ is a bad pair, there is a nonempty set of edges $S$ incident with both $v$ and $f$. If $S$ contains both primal and dual edges, both $v$ and $f$ are mixed. If all edges of $S$ are primal then by (ii) one of $v$ or $f$ must contain a dual edge, and so is mixed. A similar argument applies if all edges of $S$ are dual, and hence (i) holds.

Before completing the proof, we analyze the pairs discussed in (iii). Consider $\left(v_{i}^{\prime}, f_{j}^{\prime}\right)$ in $G^{D}$ with $v_{i}^{\prime} \in V_{\mathrm{ppv}}^{\prime}$ and $f_{j}^{\prime} \in F_{\mathrm{ppf}}^{\prime}$. Then $C_{i}^{\prime}=C_{v}$ and $A_{j}^{\prime}=A_{f}$ for some pair $(v, f)$ in $G$ where $v$ and $f$ are both primal-pure. Conversely, every pair $(v, f)$ in $G$ where $v$ and $f$ are both primal-pure corresponds to a pair $\left(v_{i}^{\prime}, f_{j}^{\prime}\right)$ in $G^{D}$ with $v_{i}^{\prime} \in V_{\mathrm{ppv}}^{\prime}$ and $f_{j}^{\prime} \in F_{\mathrm{ppf}}^{\prime}$. Moreover, $\left(v_{i}^{\prime}, f_{j}^{\prime}\right)$ is bad in $G^{D} \Leftrightarrow C_{i}^{\prime}$ and $A_{j}^{\prime}$ intersect in two or more vertices $\Leftrightarrow C_{v}$ and $A_{f}$ intersect in two or more vertices $\Leftrightarrow(v, f)$ is bad in $G$. Similarly, there is a one-to-one correspondence between pairs $\left(v_{i}^{\prime}, f_{j}^{\prime}\right)$ in $G^{D}$ with $v_{i}^{\prime} \in V_{\mathrm{dpf}}^{\prime}$ and $f_{j}^{\prime} \in F_{\mathrm{dpv}}^{\prime}$ and pairs $(v, f)$ in $G$ where $v$ and $f$ are both dual-pure, and one pair is bad if and only if the other is.

The contrapositive of (i) says: (i') every pair $(v, f)$ in $G$ with both $v$ and $f$ pure is good. We prove that $\left(\mathrm{i}^{\prime}\right) \Leftrightarrow$ (iii). First suppose that (i') holds. From above, every pair $\left(v_{i}^{\prime}, f_{j}^{\prime}\right)$ in $G^{D}$ mentioned in (iii) corresponds to a pair $(v, f)$ in $G$ where both $v$ and $f$ are pure, so that $(v, f)$ is good by $\left(\mathrm{i}^{\prime}\right)$. From above, $\left(v_{i}^{\prime}, f_{j}^{\prime}\right)$ is also good. So (iii) holds. Now suppose that (iii) holds. From above, this means that every pair $(v, f)$ in $G$ where 
$v$ and $f$ are both primal-pure, or $v$ and $f$ are both dual-pure, is good. But there are no bad pairs $(v, f)$ where $v$ is primal-pure and $f$ is dual-pure, or vice versa (these are the ' $\mathrm{X}$ ' situations in Table 1). Hence, $\left(\mathrm{i}^{\prime}\right.$ ) holds.

Next we prove a result that will be used for our global condition, so called because it deals with the largescale structure of the division between primal and dual edges in $G$. That is what determines the elements of $V_{\mathrm{tdb}}^{\prime}$ and $F_{\mathrm{tpb}}^{\prime}$. Before stating it, we need a formalism describing how a partition of the edges of a graph embedding into two parts divides up a surface into two parts.

The separation graph $Z$ for a set of edges $D$ in a graph embedding $G$ is obtained from the barycentric subdivision $S$ as follows. Each triangle $t$ of $S$ contains one vertex $u_{e} \in U_{1}$, corresponding to $e \in E(G)$. We colour $t$ red if $e \in D$, and blue if $e \notin D$. We then take the subgraph of $S$ induced by the edges with a red triangle on one side, and a blue triangle on the other side. Since all triangles incident with a vertex of $U_{1}$ are the same colour, $V(Z) \subseteq U_{0} \cup U_{2}$; its elements correspond to vertices or faces of $G$ that have edges of both $D$ and $E(G)-D$ incident with them. Thus, $Z$ is actually a subgraph of the radial graph.

When $D$ is a set of edges that we intend to dualize, we call $Z$ the primal/dual separation graph. Informally, $Z$ partitions the surface into two (closed) regions, $\Sigma_{D}$ containing dual edges of $S$ (union of closures of red faces of $S$ ) and $\Sigma_{P}$ containing primal edges (union of closures of blue faces of $S$ ); this gives a proper 2-facecolouring of $Z$. Note that the totally dual and primal subspaces satisfy $T_{D} \subseteq \Sigma_{D}$ and $T_{P} \subseteq \Sigma_{P}$. Basically, $\Sigma_{D}$ and $\Sigma_{P}$ provide extensions of $T_{D}$ and $T_{P}$, respectively, into the mixed faces of $G$ so that all of $\Sigma$ is covered. Since $Z$ is a subgraph of the radial graph, each edge of $Z$ corresponds to a corner, $y \in V(K)$, which can be thought of as its midpoint. These corners are precisely those with a dual edge on one side and a primal edge on the other side, namely the mixed vertices of $K$.

The global condition is partly expressed in terms of a Petrie dual (which has also been called the skew, as in [4, 15]). Besides the representation of Petrie dual discussed earlier (toggling the signatures of edges in a rotation system/edge signature representation), the Petrie dual $G^{\times}$of a graph embedding $G$ can be constructed directly from the embedding. The underlying graph is the same as for $G$. The boundaries of the faces of $G^{\times}$are determined by Petrie walks in $G$. To find a Petrie walk we can apply the usual facetracing algorithm, where we stay to one side of an edge as we move along it, but cross over every edge at its midpoint. In other words, we can travel along the edges, maintaining a local orientation, turning left and right at alternate vertices (so Petrie walks are sometimes called zigzag or left-right walks). We continue until our walk repeats. In this way we can find a double-covering of the edges by closed walks, discard all original faces, and glue a disk onto each Petrie walk to obtain $G^{\times}$.

Lemma 3.2 (Global incidences). (a) There is a one-to-one correspondence between $V_{\mathrm{tdb}}^{\prime} \cup F_{\mathrm{tpb}}^{\prime}$ and faces of $Z^{\times}$, the Petrie dual of the primal/dual separation graph. The division of $F\left(Z^{\times}\right)$into faces corresponding to $V_{\mathrm{tdb}}^{\prime}$ and to $F_{\mathrm{tpb}}^{\prime}$ is a 2 -face-colouring of $Z^{\times}$.

(b) Every incidence between a vertex $v_{i}^{\prime} \in V_{\mathrm{tdb}}^{\prime}$ and a face $f_{j}^{\prime} \in F_{\mathrm{tpb}}^{\prime}$ of $G^{D}$, at a corner $y$, corresponds to one of the following two situations.

(i) Cycles $C_{i}^{\prime}$ and $A_{j}^{\prime}$ meet at a mixed corner $y \in V(K)$. Then $C_{i}^{\prime}$ and $A_{j}^{\prime}$ cross at $y$. Moreover, this occurs precisely when the faces of $Z^{\times}$corresponding to $v_{i}^{\prime}$ and $f_{j}^{\prime}$ share the edge of $Z$ corresponding to $y$.

(ii) Cycles $C_{i}^{\prime}$ and $A_{j}^{\prime}$ meet at a 0-pure corner $y \in V(K)$. Then $C_{i}^{\prime}$ and $A_{j}^{\prime}$ are tangential (do not cross) at $y$.

In either case, both the vertex $\nu(y)$ and face $\phi(y)$ of $G$ associated with $y$ are mixed.

Proof. To prove (a) and (b)(i), we need to combine the separation graph and the corner graph. Recall that each vertex $y$ of the corner graph can be considered as the midpoint of the edge $\nu(y) \phi(y)$ of the radial graph, and that the separation graph is a subgraph of the radial graph. Therefore, we take $L$ to be the graph obtained by subdividing every edge of the radial graph with a vertex of $K$, then taking the union of the result with $K$. Thus, $V(L)=V(G) \cup U_{2} \cup V(K)$. In addition to the edges of $K$, for every $y \in V(K) L$ has a path $\nu(y) y \phi(y)$ of length 2. If we subdivide every edge of $Z$ to form $Z^{+}$, then $Z^{+}$is a subgraph of $L$. The new vertices created by subdivision are precisely the mixed vertices of $K$.

First we consider $v_{i}^{\prime} \in V_{\mathrm{tdb}}^{\prime}$ and the corresponding cycle $C_{i}^{\prime}$ in $K$. Since $v_{i}^{\prime} \notin V_{\mathrm{ppv}}^{\prime} \cup V_{\mathrm{dpf}}^{\prime}, C_{i}^{\prime}$ contains edges of both $A_{D}$ and $C_{P}$. So let us begin following $C_{i}^{\prime}$ at a vertex $y_{0}^{-}$with an incident edge of both $A_{D}$ and $C_{P}$ in $C_{i}^{\prime}$, leaving by the edge of $A_{D}$. Remember that $C_{i}^{\prime}$ is a boundary cycle of $\Delta$. Note that $y_{0}^{-}$is a mixed vertex of $K$. We follow edges of $A_{f_{0}} \cap Q_{D}$ where $f_{0}=\phi\left(y_{0}^{-}\right) \in F(G)$, passing through dual vertices of $K$, until we 
reach a mixed vertex $y_{0}^{+}$, still with $\phi\left(y_{0}^{+}\right)=f_{0}$. At that point we begin following edges of $C_{v_{0}} \cap Q_{P}$, where $v_{0}=\nu\left(y_{0}^{+}\right)$, passing through primal vertices of $K$, until we reach a mixed vertex $y_{1}^{-}$, still with $\nu\left(y_{1}^{-}\right)=v_{0}$. Then we follow edges of $A_{f_{1}} \cap Q_{D}$ for the new face $f_{1}=\phi\left(y_{1}^{-}\right) \in F(G)$, and so on. We continue in this way, alternating between following paths in $A_{f_{h}} \cap Q_{D}$ for faces $f_{h}=f_{0}, f_{1}, \ldots$ and paths in $C_{v_{h}} \cap Q_{P}$ for vertices $v_{h}=v_{0}, v_{1}, \ldots$. We stop when $y_{m}^{-}=y_{0}^{-}$for some $m \geq 1$, and our cycle $C_{i}^{\prime}$ has closed.

We now homotopically shift $C_{i}^{\prime}$ to obtain a new closed walk $\zeta^{+}\left(C_{i}^{\prime}\right)$ in $L$, as follows. Orient $C_{i}^{\prime}$ in the direction we traversed it above, $y_{0}^{-} \ldots y_{0}^{+} \ldots y_{1}^{-} \ldots$ Let $C_{i}^{\prime}\left[y, y^{\prime}\right]$ be the segment of $C_{i}^{\prime}$ from some $y$ to some $y^{\prime}$ in this direction (if $y=y^{\prime}$ this is a single vertex). In the segment $C_{i}^{\prime}\left[y_{h}^{-}, y_{h}^{+}\right] \subseteq A_{f_{i}} \cap Q_{D}$ every vertex $y$ has $\phi(y)=f_{h}$, so we can homotopically shift this to the path $y_{h}^{-} u_{f_{h}} y_{h}^{+}$, for $h=0,1,2, \ldots, m-1$. Similarly, in each segment $C_{i}^{\prime}\left[y_{h}^{+}, y_{h+1}^{-}\right] \subseteq C_{v_{i}}^{\prime} \cap Q_{P}$ (subscripts on $y_{h}^{ \pm}$interpreted modulo $m$ ) every vertex $y$ has $\nu(y)=v_{h}$, so we can homotopically shift this to $y_{h}^{+} v_{h} y_{h+1}^{-}$, for $h=0,1,2, \ldots, m-1$. Therefore, $\zeta^{+}\left(C_{i}^{\prime}\right)=y_{0}^{-} u_{f_{0}} y_{0}^{+} v_{0} y_{1}^{-} u_{f_{1}} y_{1}^{+} v_{1} y_{2}^{-} \ldots v_{m-1}\left(y_{m}^{-}=y_{0}^{-}\right)$.

Since each $y_{h}^{ \pm}$is a mixed vertex of $K$, each path $u_{f_{h}} y_{h}^{+} v_{h}$ and each path $v_{h} y_{h+1}^{-} u_{f_{h+1}}$ is just a subdivided edge of $Z$. Skipping the vertices $y_{h}^{ \pm}$of degree 2 in $Z^{+}, \zeta^{+}\left(C_{i}^{\prime}\right)$ becomes a closed walk in $Z$, namely $\zeta\left(C_{i}^{\prime}\right)=$ $u_{f_{0}} v_{0} u_{f_{1}} v_{1} \ldots v_{m-1} u_{f_{0}}$. We notice that this walk turns to follow $\partial \Sigma_{D}$ at each $u_{f_{h}}$, and to follow $\partial \Sigma_{P}$ at each $v_{h}$. In other words, it turns alternately left and right for some local orientation, and is a Petrie walk in $Z$, as required.

In a similar way, there is a Petrie walk $\zeta\left(A_{j}^{\prime}\right)$ in $Z$ for each $f_{j}^{\prime} \in F_{\mathrm{tpb}}^{\prime}$, which turns to follow $\partial \Sigma_{D}$ at each vertex in $V(G)$, and to follow $\partial \Sigma_{P}$ at each vertex in $U_{2}$.

So the $\zeta\left(C_{i}^{\prime}\right)$ and $\zeta\left(A_{j}^{\prime}\right)$ form the faces of $Z^{\times}$. Every mixed corner of $K$ has both some $C_{i}^{\prime}$ for $v_{i}^{\prime} \in V_{\mathrm{tdb}}^{\prime}$ and some $A_{j}^{\prime}$ for $f_{j}^{\prime} \in F_{\mathrm{tpb}}^{\prime}$ passing through it, so every edge of $Z$, or $Z^{\times}$, belongs to some $\zeta\left(C_{i}^{\prime}\right)$ and some $\zeta\left(A_{j}^{\prime}\right)$. Hence, each type of face in $Z^{\times}$covers each edge of $Z^{\times}$exactly once, so we have the required one-to-one correspondence and 2 -face-colouring.

(b) Each incidence of $v_{i}^{\prime}$ and $f_{j}^{\prime}$ is represented by a vertex $y \in V(K)$ common to $C_{i}^{\prime}$ and $A_{j}^{\prime}$. Suppose $y$ corresponds to $v \in V(G), f \in F(G)$, and ends of $e, e^{\prime} \in E(G)$. Then the faces around $y$ occur in the order $c_{v}, q_{e}, a_{f}, q_{e^{\prime}}$.

First suppose $y$ is mixed. Then $e \in D$ and $e^{\prime} \in P$ (or vice versa), so $v$ and $f$ are mixed. The edges incident with $y$ in $K$ then belong to $C_{P}, C_{D}, A_{D}$ and $A_{P}$ in that order (or its reverse). Now $C_{i}^{\prime} \subseteq C_{P} \cup A_{D}$ and $A_{j}^{\prime} \subseteq C_{D} \cup A_{P}$, which therefore must cross at $y$. Also, our argument for (a), above, shows that if $y$ is mixed then the corresponding faces $\zeta\left(C_{i}^{\prime}\right)$ and $\zeta\left(A_{j}^{\prime}\right)$ of $Z^{\times}$share the edge $\nu(y) u_{\phi(y)}$ of $Z^{\times}$. Conversely, if $\zeta\left(C_{i}^{\prime}\right)$ and $\zeta\left(A_{j}^{\prime}\right)$ share an edge of $Z^{\times}$, or equivalently of $Z$, then they both contain the mixed vertex of $K$ with which this edge is subdivided in $Z^{+}$.

So now suppose $y$ is pure. Assume first that $y$ is primal-pure. Then $e, e^{\prime} \in P$. The edges incident with $y$ in $K$ are therefore $d_{1}, d_{2} \in C_{v} \cap Q_{P} \subseteq C_{P}$ followed by $d_{3}, d_{4} \in A_{f} \cap Q_{P} \subseteq A_{P}$ in that order. Therefore, $C_{i}^{\prime} \subseteq C_{P} \cup A_{D}$ and $A_{j}^{\prime} \subseteq C_{D} \cup A_{P}$ do not cross at $y$. If $v$ is pure, then since $C_{v}$ and $C_{i}^{\prime}$ both contain $d_{1}, \bar{d}_{2} \in C_{P}, v$ is primal-pure, and then $C_{i}^{\prime}=C_{v}$ so that $v_{i}^{\prime} \in V_{\mathrm{ppv}}^{\prime}$, contradicting $v_{i}^{\prime} \in V_{\mathrm{tdb}}^{\prime}$. So $v$ is mixed. A similar argument shows that $f$ is also mixed. Therefore $y$ is 0-pure. Similar arguments again apply if $y$ is dual-pure.

Therefore, the cases where $y$ is mixed or pure correspond exactly to parts (i) and (ii) as stated. In both cases $v=\nu(y)$ and $f=\phi(y)$ are mixed.

Condition (b)(i) here is quite nice, being expressible in terms of the primal/dual separation graph, which reflects global structure. Condition (b)(ii) does not seem to be expressible in such a succinct way. However, it does restrict which corners we need to examine: we do not need to examine 2-pure or 1-pure corners for vertex/face pairs of this type.

Note that if $Z$ has a vertex $z$ of degree two, then situation (b)(i) occurs on both edges of $Z^{\times}$incident with $z$ for the two faces of $Z^{\times}$that pass through $z$, and hence the corresponding vertex/face pair in $G^{D}$ is bad. This happens if there is a vertex or face of $G$ that is incident exactly once with a region of $\Sigma_{P}$ and exactly once with a region of $\Sigma_{D}$. For example, dualizing a single link (non-loop) or colink (edge incident with two distinct faces) always creates a bad pair.

Finally we prove a result that we will use for our midrange condition, so called because it deals with the incidence of local structures (faces or vertices in $G^{D}$ coming from pure faces or vertices in $G$ ) with large-scale structures (faces or vertices in $G^{D}$ coming from totally dual or primal regions). Like condition (b)(ii) in Lemma 3.2 it does not seem to be expressible in a succinct way, but it does provide some information about where we should focus our attention. 
Lemma 3.3 (Midrange incidences). Suppose we have an incidence in $G^{D}$ between $v_{i}^{\prime}$ and $f_{j}^{\prime}$ corresponding to a corner $y \in V(K)$ where $C_{i}^{\prime}$ and $A_{j}^{\prime}$ meet. Let $\nu(y)=v$ and $\phi(y)=f$.

(a) If $v_{i}^{\prime} \in V_{\mathrm{tdb}}^{\prime}$ and $f_{j}^{\prime} \in F_{\mathrm{dpv}}^{\prime}$, then $f_{j}^{\prime}$ corresponds to $v$ and $f$ is mixed.

(b) If $v_{i}^{\prime} \in V_{\mathrm{tdb}}^{\prime}$ and $f_{j}^{\prime} \in F_{\mathrm{ppf}}^{\prime}$, then $f_{j}^{\prime}$ corresponds to $f$ and $v$ is mixed.

(c) If $f_{j}^{\prime} \in F_{\mathrm{tpb}}^{\prime}$ and $v_{i}^{\prime} \in V_{\mathrm{ppv}}^{\prime}$, then $v_{i}^{\prime}$ corresponds to $v$ and $f$ is mixed.

(d) If $f_{j}^{\prime} \in F_{\mathrm{tpb}}^{\prime}$ and $v_{i}^{\prime} \in V_{\mathrm{dpf}}^{\prime}$, then $v_{i}^{\prime}$ corresponds to $f$ and $v$ is mixed.

In all of these cases $y$ is 1-pure.

Proof. We prove (a); the others are similar. Since $f_{j}^{\prime} \in F_{\mathrm{dpv}}^{\prime}$, then $A_{j}^{\prime}=C_{u}$ for some $u \in V(G)$. But $A_{j}^{\prime}=C_{u}$ shares $y$ with $C_{v}$, so $u=v$, and $v$ is dual-pure. Now if $f$ is pure then it is also dual-pure, since it contains two edge-ends incident with $v$, so then $A_{f}=C_{k}^{\prime}$ for some $k$. But $C_{k}^{\prime}$ shares $y$ with $C_{i}^{\prime}$, so $k=i$ and $A_{f}=C_{i}^{\prime}$, giving $v_{i}^{\prime} \in V_{\mathrm{dpf}}^{\prime}$, a contradiction. Hence, $f$ is mixed, and since $v$ is pure, $y$ is 1-pure.

Essentially, the local incidences occur at 2-pure corners, the global incidences occur at mixed or 0-pure corners, and midrange incidences occur at 1-pure corners. This means that for any given pair of cycles $C_{i}^{\prime}$ and $A_{j}^{\prime}$, we can restrict our attention only to a certain set of corners. If some types of corners do not occur, then it may greatly simplify the checking of whether $G^{D}$ is closed 2-cell or not.

Now we can state our main theorem, which follows immediately from Lemmas 3.1, 3.2 and 3.3 . Call a pure vertex of $G$ exposed if it has more than one incidence with mixed faces of $G$ (in other words, more than one corner at that vertex is 1-pure). Similarly, call a pure face of $G$ exposed if it has more than one incidence with mixed vertices of $G$ (in other words, more than one corner of that face is 1-pure).

Theorem 3.4. Suppose $G$ is a graph embedding with at least two edges, and $D \subseteq E(G)$. Then the partial dual $G^{D}$ is closed 2-cell if and only if all of the following conditions hold.

(LC) For every bad vertex/face pair $(v, f)$ in $G$, at least one of $v$ or $f$ is mixed (or, equivalently, $\delta_{G} v \cup \partial_{G} f$ contains both a primal and a dual edge).

(MC) For every exposed primal-pure (dual-pure) vertex $v$ of $G$, every boundary walk of a totally primal (dual) region passes through a corner at $v$ at most once. Also, for every exposed dual-pure (primal-pure) face $f$ of $G$, every boundary walk of a totally primal (dual) region passes through a corner of $f$ at most once.

(GC) The embedding $Z^{\times}$, the Petrie dual of the primal/dual separation graph of $G$, has no two faces that share two or more edges. Also, for each 0-pure corner, with associated vertex $v_{i}^{\prime}$ and $f_{j}^{\prime}$ in $G^{D}$, the faces of $Z^{\times}$associated with $v_{i}^{\prime}$ and $f_{j}^{\prime}$ do not share an edge in $Z^{\times}$. Nor is there another 0-pure corner that is associated with the same $v_{i}^{\prime}$ and $f_{j}^{\prime}$ in $G^{D}$.

One situation where we can check whether an embedding is closed 2-cell in a nice way is covered by the following corollary.

Corollary 3.5. Suppose $G$ is a closed 2 -cell graph embedding with at least two edges, and $D \subseteq E(G)$. Suppose that for this $D$ there are no exposed pure vertices or faces, and no 0-pure corners. Then $G^{D}$ is closed 2 -cell if and only if $Z^{\times}$, the Petrie dual of the primal/dual separation graph, has no two faces that share two or more edges.

To convince the reader that this corollary, and indeed our main theorem, is useful, we now provide an example where Corollary 3.5 can be used to demonstrate that a nontrivial partial dual is closed 2-cell. In Figure 6 we see an example of a closed 2-cell embedding on the torus (represented in the standard way as a rectangle, where we consider the top and bottom sides to be identified and the left and right sides to be identified). It is made up of eight diamonds (copies of $K_{4}-e$ ). We dualize the four diamonds oriented vertically (dual edges are denoted by wavy curves, as usual). Then the pure vertices, pure faces, and totally primal or dual boundary walks are all similar to those shown in the figure. Every pure vertex touches exactly one mixed face, and every pure face touches exactly one mixed vertex, so there are no exposed pure vertices or faces. Every corner is mixed (between two diamonds), 2-pure (four central corners inside each diamond), or 1-pure (remaining corners); there are no 0-pure corners. Therefore, the only thing we need to check is that the Petrie dual of the primal/dual separation graph does not have two faces sharing two edges. Equivalently, we just need to show that boundary walks of a totally primal region and of a totally dual region never intersect more than once. But the boundary walks of totally primal regions run horizontally across our figure, and those of totally dual regions run vertically, and so they always meet exactly once. So we know that the partial dual $G^{D}$ constructed in this situation is closed 2-cell. 


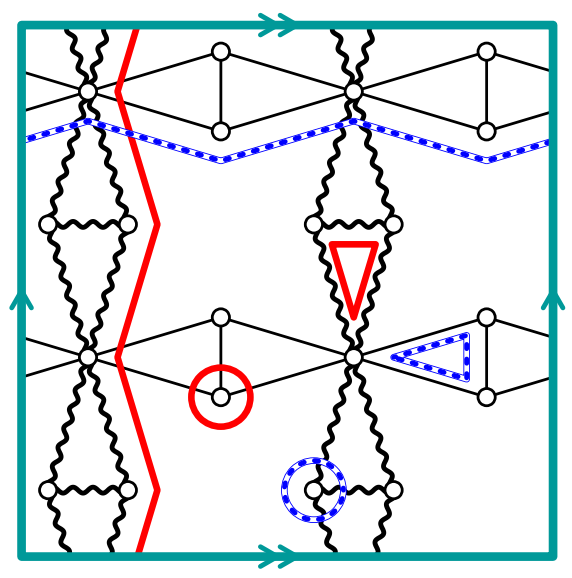

Figure 6: A closed 2-cell nontrivial partial dual

We can also provide the reader with another example, also for an embedding on the torus, where a partial dual is guaranteed not to be closed 2-cell. It is not difficult to construct a toroidal graph embedding with primal and dual edges so that the primal/dual separation graph $Z$ forms a 2 -face-colourable grid, i.e., a standard toroidal embedding of the cartesian product $C_{2 m} \times C_{2 n}$ for some $m, n \geq 2$, represented in the usual rectangular model of the torus by horizontal and vertical lines. The Petrie walks in the primal/dual separation graph are then stair-stepping walks that go up and right, or down and right. The two Petrie walks sharing an edge go in different directions. In the usual representation of the homotopy group of the torus as $\mathbb{Z} \oplus \mathbb{Z}$, the up-and-right walks will have type $(a, b)$, while the down-and-right walks will have type $(a,-b)$, for some $a, b \geq 1$. It is well known that two closed curves of homotopy types $(a, b)$ and $(c, d)$ in the torus must intersect at least $|a d-b c|$ times, so our walks must intersect at least $2 a b \geq 2$ times. If stair-stepping walks going in opposite directions intersect, they must do so along an edge. Therefore, any two Petrie walks going in different directions share at least two edges, and so the partial dual cannot be closed 2-cell.

We conclude this section by discussing some graph embeddings that have no partial dual that is closed 2-cell. Our condition involves separability.

We have mentioned that a graph with a closed 2-cell embedding must be nonseparable. In other words, a separable graph does not have a closed 2-cell embedding. However, a separable graph may have a partial dual that is closed 2-cell. We can see this by working backwards. Suppose we take a closed 2-cell embedding $G$, and let $D$ be the edges of a spanning tree in $G$. Then the region $\Delta$ is just a fattened version of the tree formed by $D$, and so has a single boundary component. Therefore, by Proposition $2.2, H=G^{D}$ has a single vertex, and every edge is a loop, so $H$ is separable (in fact, every edge can be separated from all the other edges). But $H^{D}=G$, which is closed 2-cell.

So we need a stronger condition than just separability to guarantee that a graph embedding $G$ has no partial dual that is closed 2-cell. Our reasoning uses gems, and we need the following standard result about proper 3-edge-colourings of cubic graphs.

Parity Lemma ([2, 8]). Suppose $S$ is an edge cut in a properly 3-edge-coloured cubic graph. Let $S_{c}$ be the set of all edges in $S$ of colour $c$. Then $|S| \equiv\left|S_{c}\right|(\bmod 2)$.

Lemma 3.6. Suppose $M$ is a gem. Then $M$ is 2-edge-connected, every 2-edge cut in $M$ consists of two edges of the same colour, if $|V(M)|>4$ and $M$ has a 2-edge cut then it has one consisting of two yellow edges, and if $M$ has no 2-edge cut then it is cyclically-4-edge-connected.

Proof. An edge cut of a single edge cannot satisfy the Parity Lemma for all colours, so any edge cut must have at least two edges. Moreover, if there is a 2-edge cut, then the Parity Lemma implies that both edges are the same colour.

Assume there is a red 2-edge cut $\left\{g_{1}, g_{2}\right\}$ and no yellow 2-edge cut. Suppose $g_{1}$ joins $x_{1}$ and $x_{1}^{\prime}$. The red-blue bigon containing $g_{1}$ must also contain $g_{2}$, and must be a cycle $\left(x_{1} x_{1}^{\prime} x_{2}^{\prime} x_{2}\right)$ where $g_{2}$ joins $x_{2}$ and $x_{2}^{\prime}$. Then the two yellow edges incident with $x_{1}$ and $x_{2}$ form a 2-edge cut, unless they are the same edge. By the same reasoning, the two yellow edges incident with $x_{1}^{\prime}$ and $x_{2}^{\prime}$ form a 2-edge cut unless they are the same edge. But then $|V(M)|=4$, a contradiction. A similar argument applies to a blue 2-edge cut. 
Suppose $M$ has no 2-edge cut, but has a 3-edge cut $S=\left\{g_{1}, g_{2}, g_{3}\right\}$. Then $g_{1}, g_{2}, g_{3}$ are different colours by the Parity Lemma. Suppose $g_{1}$ is red and $g_{2}$ is blue. The red-blue bigon containing $g_{1}$ must use $g_{2}$, which means that $g_{1}$ and $g_{2}$ share a vertex $x$. Let $g_{4}$ be the yellow edge incident with $x$. If $g_{4} \neq g_{3}$, then $\left\{g_{3}, g_{4}\right\}$ is a 2-edge cut, which is impossible, so $g_{4}=g_{3}$ and all edges of $S$ are incident with the same vertex. This is true for all 3-edge cuts, so $M$ is cyclically-4-edge-connected.

We can interpret 2-edge cuts in the gem $M$ in terms of the graph embedding $G$. Suppose $S=\left\{g_{1}, g_{2}\right\}$ is a 2-edge cut that separates $M$ into two components $M_{1}$ and $M_{2}$. By Lemma 3.6 both edges of $S$ are the same colour.

If $S$ is yellow, then there is a red-yellow bigon $C_{v}$ and a blue-yellow bigon $A_{f}$ that both use $g_{1}$ and $g_{2}$. From our discussion at the beginning of this section, this means that $(v, f)$ is a bad vertex/face pair in $G$. Taking the embedding of $M$ corresponding to, and in the same surface as, the embedding $G$, we can form a simple closed curve $\Psi$ intersecting each of $g_{1}$ and $g_{2}$ exactly once, contained in $\overline{c_{v}} \cup \overline{a_{f}}$ from $M$, and also contained in $f \cup v$ from $G$. Then $\Psi$ separates the surface into two components, one containing $M_{1}$ and the other containing $M_{2}$. Deleting $v$ and $f$ deletes $\Psi$, so it also disconnects the surface. We call $(v, f)$ a separating vertex/face pair.

If $S$ is red, then there is a red-yellow bigon $C_{v}$ and a red-blue bigon $Q_{e}$ that both use $g_{1}$ and $g_{2}$. In $G$ this means that the edge $e$ is a loop at $v$. In a manner similar to the previous paragraph, we can construct a simple closed curve $\Psi$ contained in $\overline{c_{v}} \cup \overline{q_{e}}$, and also passing through $v$, which separates $\Sigma$. In this case $\Psi$ is homotopic (with base point $v$ ) to $e$, and so $e$ is a separating simple closed curve. We call $e$ a separating loop.

If $S$ is blue, then we have the dual situation to when $S$ is red. In this case the edge $e^{*}$ in $G^{*}$ dual to $e$ is a separating loop, so we say $e$ is a separating coloop: $e$ is an edge with the same face on both sides that is also a cutedge in $G$.

Putting these together with Lemma 3.6 we obtain the following sufficient condition for a graph embedding to have no closed 2-cell partial duals.

Theorem 3.7. If a graph embedding $G$ with at least two edges has a separating vertex/face pair, a separating loop, or a separating coloop, then every partial dual of $G$ has a separating vertex/face pair. Hence, no partial dual of $G$ is closed 2-cell.

Proof. The hypothesis lists the situations under which the gem of $G$ has a 2-edge-cut. Then by Lemma 3.6 it has a yellow 2-edge cut, which remains a yellow 2-edge cut in the gem of every partial dual. Hence every partial dual has a separating vertex/face pair, which is a bad pair, so the partial dual is not closed 2-cell.

Question 3.8. For a graph embedding to have a closed 2-cell partial dual, we have seen that it is necessary for the gem to have no 2-edge-cut. It is natural to ask whether this is sufficient. In other words, if $G$ is a graph embedding with no separating vertex/face pair, does there exist a closed 2-cell partial dual of $G$ ?

To conclude, we have developed some techniques in this paper which allow us to give some conditions under which a partial dual is or is not closed 2-cell. One goal would natually be to try to find ways to use this to construct closed 2-cell embeddings of some classes of graph, to address the Strong Embedding and Cycle Double Cover Conjectures. The difficulty is that partial duality does not preserve the underlying graph (unlike, for example, partial Petrie duality). It therefore seems difficult to incorporate partial duality into an attack on these problems.

\section{Acknowledgement}

The authors thank both referees for helpful comments that improved the presentation of the paper.

\section{References}

[1] D.W. Barnette, Generating closed 2-cell embeddings in the torus and the projective plane, Discrete Comput. Geom. 2 (1987) 233-247.

[2] D. Blanuša, Problem četiriju boja (The problem of four colors), Hrvatsko Prirodoslovno Društvo Glasnik Mat-Fiz. Astr. Ser. II 1 (1946) 31-42.

[3] B. Bollobás and O. Riordan, A polynomial of graphs on surfaces, Math. Ann. 323 (2002) 81-96. 
[4] C. P. Bonnington and C. H. C. Little, The foundations of topological graph theory, Springer, New York, 1995.

[5] R. Bradford, C. Butler and S. Chmutov, Arrow ribbon graphs, J. Knot Theory Ramifications 21 no. 13 (2012) 1240002 (16 pages).

[6] S. Chmutov, Generalized duality for graphs on surfaces and the signed Bollobás-Riordan polynomial, $J$. Combin. Theory Ser. B 99 (2009) 617-638.

[7] S. Chmutov and F. Vignes-Tourneret, Partial duality of hypermaps, arXiv:1409.0632 1.

[8] B. Descartes, Network-colourings. Math. Gaz. 32 (1948) 67-69.

[9] M. Ellingham, Chmutov's generalized duality and the gem representation of embedded graphs, Abstract 1077-05-1582 (Joint Mathematics Meetings, Boston, MA, 2012) Abstracts Amer. Math. Soc. 33 no. 1 (2012) 36 [available online at http://jointmathematicsmeetings.org/amsmtgs/2138_abstracts/ 1077-05-1582.pdf.

[10] M. N. Ellingham and Xiaoya Zha, Orientable embeddings and orientable cycle double covers of projective-planar graphs, European J. Combinatorics 32 (2011) 495-509.

[11] J. A. Ellis-Monaghan and I. Moffatt, Twisted duality for embedded graphs, Trans. Amer. Math. Soc. 364 (2012) 1529-1569.

[12] J. A. Ellis-Monaghan and I. Moffatt, Graphs on surfaces: dualities, polynomials, and knots, SpringerBriefs in Mathematics, Springer, New York, 2013.

[13] G. Haggard, Edmonds characterization of disc embeddings, Proc. 8th Southeastern Conf. on Combinatorics, Graph Theory and Computing (Baton Rouge, Louisiana, 1977), Congr. Numer. 19 (1977) 291-302.

[14] J. L. Gross and T. W. Tucker, Topological graph theory, Dover, Mineola, New York, 2001.

[15] S. Lins, Graph-encoded maps, J. Combin. Theory Ser. B 32 (1982) 171-181.

[16] B. Mohar and C. Thomassen, Graphs on surfaces, Johns Hopkins University Press, Baltimore, 2001.

[17] N. Robertson, Pentagon-generated trivalent graphs with girth 5, Canad. J. Math. 23 (1971) 36-47.

[18] P. D. Seymour, Sums of circuits, in Graph theory and related topics (ed. J. A. Bondy and U. R. S. Murty), Academic Press, New York, 1979, pp. 341-355.

[19] G. Szekeres, Polyhedral decompositions of cubic graphs, Bull. Austral. Math. Soc. 8 (1973) 367-387. 